\title{
Guillain-Barre Syndrome and Pericarditis: A Rare Association
}

\author{
Ersel Dag ${ }^{\mathrm{a}, \mathrm{c}}$, Burcu Gokce Cokal ${ }^{\mathrm{a}}$, Murat Tulmac ${ }^{\mathrm{b}}$, Yakup Turkel ${ }^{\mathrm{a}}$, Vedat Simsek ${ }^{\mathrm{b}}$, Betul Acar $^{\mathrm{a}}$
}

\begin{abstract}
Guillain-Barre syndrome (GBS) is an acute inflammatory polyradiculoneuropathy and often develops due to the immune reaction that is related with an event such as an acute infection that is just before the syndrome. Up to date there are only two reported cases of pericarditis that are associated with GBS in the literature. We here presented a 52-year-old male patient of who numbness and loss of power developed at distal extremities after an upper respiratory tract infection. The patient was diagnosed as GBS with pericarditis. GBS and pericarditis relationship is discussed in scope of the literature.
\end{abstract}

Keywords: Guillain-Barre syndrome; Pericarditis; Upper respiratory tract infection

\section{Introduction}

Guillain-Barre syndrome (GBS) is characterized with areflexia and progressive weakness in the extremities, and is an acute inflammatory demyelinating polyneuropathy that is reported all over the world as the most common cause of neuromuscular paralysis [1]. GBS is an autoimmune disorder that emerges as a result of a postinfectious defect at a population that is generally healthy and that does not have any systemic or autoimmune disease. This case is the third reported in the literature in terms of the association of GBS and pericarditis.

\footnotetext{
Manuscript accepted for publication August 12, 2013

${ }^{a}$ Department of Neurology, School of Medicine, Kirikkale University, Kirikkale, Turkey

${ }^{\mathrm{b}}$ Department of Cardiology, School of Medicine, Kirikkale University, Kirikkale, Turkey

${ }^{\mathrm{c} C}$ Corresponding author: Ersel Dag, Department of Neurology, School of Medicine, Kirikkale University, Kirikkale, Turkey.

Email:erseldag@yahoo.com
}

doi: http://dx.doi.org/10.4021/jmc1481w

\section{Case Report}

A 52-year-old male patient applied to our clinic with the complaint of numbness and loss of strength at the extremities for three days. In his story it is learned that the patient had upper respiratory tract infection 10 days ago. Numbness of the feet began a few days before the numbness of hands and afterwards loss of strength began to develop at the legs and arms. Any significant pathological finding was not detected at the physical examination. At the neurologic examination of the patient during the application, the patient was conscious, cooperative, and oriented. Cranial nerve examination was normal. The deep tendon reflexes (DTR) were not detected at the lower extremities while they were hypoactive in the upper extremities. There were stocking style hypoestesia at both legs. Bilateral proximal and distal upper limb muscle strength was found to be $4 / 5$ and bilateral proximal and distal lower extremity muscle strength was found to be $3 / 5$, respectively. The base skin reflexes were evaluated as flexor, bilaterally.

In electromyography (EMG) examination, with the axonal and demyelinating involvement, widespread sensory motor type polyneuropathy and loss of $\mathrm{F}$ responses was observed. Cerebrospinal fluid (CSF) was seemed to have a clear view and the pressure was found to be normal. In the microscopic examination of CSF no cells were observed, and total protein was found to be high $(144 \mathrm{mg} / \mathrm{dL}$; normal range: 15 - $45 \mathrm{mg} / \mathrm{dL}$ ).

The patient was diagnosed to have GBS and was administered $400 \mathrm{mg} / \mathrm{kg}$ per day intravenous immune globulin (IVIG) treatment for 5 days. At the 2 th day of admission the patient had the complaint of palpitations, shortness of breath, and chest pain that increases with lying on his back and that is radiating to both of the shoulders. Cardiological evaluation was normal but at the ECG examination pericardial effusion was detected, diagnosis of acute pericarditis was done and ibuprofen treatment was started.

Viral markers that were screened in order to evaluate the etiology were found to be negative. Fever and diarrhea developed during his stay at the clinic. Thus, a complete blood count, sedimentation rate, peripheral blood smear, biochem- 
istry, ASO, throat, urine, and stool culture was performed and empiric antibiotic moxifloxacin was started at a dose of $400 \mathrm{mg}$ daily IV. Culture results were negative. At the 10th day of antibiotic therapy, the therapy was stopped. Granted for a period of five days prior to at the 10th day following the discontinuation of the IVIG treatment after administration for 5 days, hypoesthesia was reduced and hypoactive DTR's at the lower extremities can be able to be taken at the neurological examination. Bilateral proximal and distal upper limb muscle strength was found to be $5 / 5$, while bilateral proximal lower extremity muscle strength was $4 / 5$ and the distal was $5 / 5$, respectively. 14 days after the application for chest pain, pericardial effusion was disappeared at the echocardiography.

\section{Discussion}

GBS and the coexistence of pericarditis have rarely been reported in the literature. In our case, this developed condition can be interpreted as the coexistence of two rare condition or complication of GBS with pericarditis. Of the GBS patients in the literature about at $2 / 3$, prior infection symptoms were detected within 3 weeks before the muscle weakness symptoms were emerged [2]. A nearly similar prodromal period can also seen at the most common form of acute pericarditis, which is known as acute idiopathic pericarditis. In our patient, similar to the findings of this study, 10 days before the occurrence of symptoms of the GBS symptoms of influenza, such as fever and runny nose emerged. This situation suggests that there seems to be a possibility that the same factor causes GBS and as well as pericarditis.

Although, in the literature many types of infections were reported to be in association with GBS, the most common causative infections are upper respiratory and gastrointestinal infections. Campylobacter jejuni, CMV, EBV, M. pneumoniae and $\mathrm{H}$. influenzae are considered as common triggering agents of GBS [3]. On the other hand, although it is controversial,in the literature, events other than the infections such as, vaccines, operations and stress are reported to be associated with GBS $[4,5]$.

In the literature, there are 2 patients that have the coexistance of GBS with pericarditis. In the case report of Kao et al, a 68-year- old female patient at which GBS was emerged after the influenzae vaccination, was diagnosed to have pericarditis based on echocardiography. The patient recovered fully within two months with plasmapheresis [6]. Komiagin et al. reported GBS and exudative pericarditis emerged after acute hepatitis $\mathrm{B}$ infection in a patient receiving hemodialysis [7].

Like the general population, due to the high probability of missing the pericarditis at the patients with GBS, pericarditis may not be so uncommon contrary to the beliefs. For this reason, GBS patients deserve a careful review for symptoms of pericarditis.

\section{Acknowledgement}

This case was presented as a poster in the 7th World Congress on Controversies in Neurology (CONy), April 11 - 14, 2013.

\section{References}

1. Alshekhlee A, Hussain Z, Sultan B, Katirji B. GuillainBarre syndrome: incidence and mortality rates in US hospitals. Neurology. 2008;70(18):1608-1613.

2. Koga M, Yuki N, Hirata K. Antecedent symptoms in Guillain-Barre syndrome: an important indicator for clinical and serological subgroups. Acta Neurol Scand. 2001;103(5):278-287.

3. Jacobs BC, Rothbarth PH, van der Meche FG, Herbrink P, Schmitz PI, de Klerk MA, van Doorn PA. The spectrum of antecedent infections in Guillain-Barre syndrome: a case-control study. Neurology. 1998;51(4):1110-1115.

4. Hughes RA, Cornblath DR. Guillain-Barre syndrome. Lancet. 2005;366(9497):1653-1666.

5. Lasky T, Terracciano GJ, Magder L, Koski CL, Ballesteros M, Nash D, Clark S, et al. The Guillain-Barre syndrome and the 1992-1993 and 1993-1994 influenza vaccines. N Engl J Med. 1998;339(25):1797-1802.

6. Kao CD, Chen JT, Lin KP, Shan DE, Wu ZA, Liao KK. Guillain-Barre syndrome coexisting with pericarditis or nephrotic syndrome after influenza vaccination. Clin Neurol Neurosurg. 2004;106(2):136-138.

7. Komiagin Iu V, Mozolevskii Iu V, Milovanov Iu S, Ermolenko VM. [Guillain-Barre syndrome and exudative pericarditis as complications of acute viral hepatitis B during programmed hemodialysis]. Klin Med (Mosk). 1990;68(11):93-95. 